\title{
Giant and Tunneling Magnetoresistance in Unconventional Collinear Antiferromagnets with Nonrelativistic Spin-Momentum Coupling
}

\author{
Libor Šmejkal, ${ }^{1,2}$ Anna Birk Hellenes $\odot,{ }^{1}$ Rafael González-Hernández, ${ }^{3}$ Jairo Sinova, ${ }^{1,2}$ and Tomas Jungwirth ${ }^{2,4}$ \\ ${ }^{1}$ Institut für Physik, Johannes Gutenberg Universität Mainz, D-55099 Mainz, Germany \\ ${ }^{2}$ Institute of Physics, Czech Academy of Sciences, Cukrovarnická 10, 16200 Praha 6, Czech Republic \\ ${ }^{3}$ Grup de Investigación en Física Aplicada, Departamento de Física, Universidad del Norte, \\ Barranquilla, Colombia \\ ${ }^{4}$ School of Physics and Astronomy, University of Nottingham, Nottingham NG7 2RD, United Kingdom
}

(Received 5 April 2021; revised 21 October 2021; accepted 13 December 2021; published 10 February 2022)

Giant and tunneling magnetoresistance are physical phenomena used for reading information in commercial spintronic devices. The effects rely on a conserved spin current passing between a reference and a sensing ferromagnetic electrode in a multilayer structure. Recently, we have proposed that these fundamental spintronic effects can be realized in unconventional collinear antiferromagnets with nonrelativistic alternating spin-momentum coupling. Here, we elaborate on the proposal by presenting archetype model mechanisms for the giant and tunneling magnetoresistance effects in multilayers composed of these unconventional collinear antiferromagnets. The models are based, respectively, on anisotropic and valley-dependent forms of the alternating spin-momentum coupling. Using first-principles calculations, we link these model mechanisms to real materials and predict an approximately $100 \%$ scale for the effects. We point out that, besides the giant or tunneling magnetoresistance detection, the alternating spin-momentum coupling can allow for magnetic excitation by the spin-transfer torque.

DOI: $10.1103 /$ PhysRevX.12.011028

\section{INTRODUCTION}

In the nonrelativistic band structure of ferromagnets, the exchange interaction induces an energy gap between spinup and spin-down states, making one spin state more populated and the other one less. This results in different Ohmic resistivities of the majority and minority spin channels and in spin-dependent density of states. The former is the basis of the giant magnetoresistance (GMR) effect in a trilayer stack comprising two ferromagnetic metal electrodes separated by a nonmagnetic metal spacer [1]. The different densities of states of the majority and minority spins then govern the tunneling magnetoresistance (TMR) in a trilayer with a tunnel barrier between the ferromagnetic electrodes [1]. In both GMR and TMR, the resistance of the stack depends on whether the ferromagnetic electrodes are magnetized parallel or antiparallel, with typically the higher-resistance state corresponding to the antiparallel configuration. The well-conserved spin currents in collinear ferromagnets, enabling the GMR or

Published by the American Physical Society under the terms of the Creative Commons Attribution 4.0 International license. Further distribution of this work must maintain attribution to the author(s) and the published article's title, journal citation, and DOI.
Subject Areas: Condensed Matter Physics, Materials Science, Spintronics
TMR readout of the magnetization reversal, can also facilitate efficient electrical switching of the magnetization via a spin-transfer torque (STT) [2]. The superior on-off characteristics of the devices with STT switching and GMR or TMR readout allowed spintronics to revolutionize the magnetic storage and memory industry $[1,3]$.

Conventionally, nonrelativistic spin-split bands are considered to be excluded in collinear antiferromagnets due to the antiparallel atomic moments in the crystal [4-9]. A characteristic example are antiferromagnets with a symmetry combining time reversal with space inversion, which results in Kramers spin degeneracy of electronic bands over the entire Brillouin zone [10]. Considering such a model antiferromagnet, a STT mechanism was theoretically proposed more than a decade ago which differs fundamentally from STT in ferromagnets [6]. It is based on transmitting a staggered spin polarization from one to the other antiferromagnet where the spin polarization and the antiferromagnetic orders in the electrodes are all commensurate [6]. This is a subtle, spin-coherent quantum-interference phenomenon relying on perfectly epitaxial commensurate multilayers $[6,11,12]$. Similarly delicate were the proposed GMR and TMR effects in these antiferromagnetic structures [11], which might explain why a nonrelativistic spintronics concept based on the conventional collinear antiferromagnets with spin-degenerate bands has not been experimentally viable. 
(a)

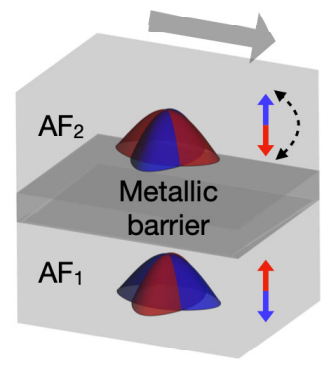

(b)

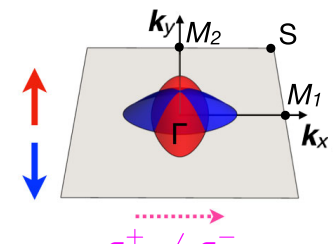

$\sigma_{x x}^{+} \neq \sigma_{x x}^{-} \quad$ Anisotropic

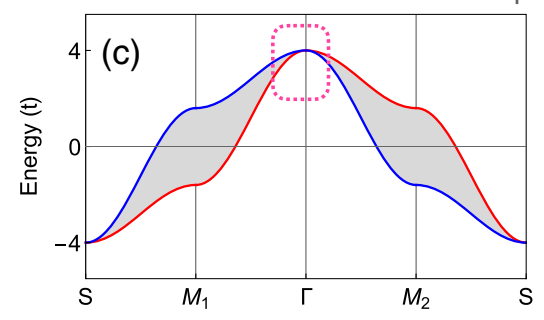

(d)

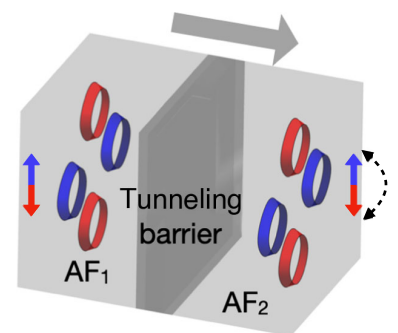

(e)

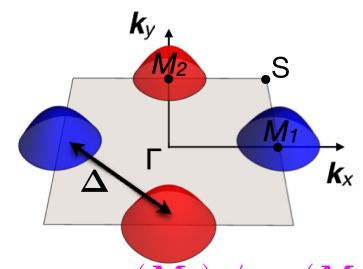

Valley-dependent $n_{+}\left(\boldsymbol{M}_{1}\right) \neq n_{-}\left(\boldsymbol{M}_{1}\right)$

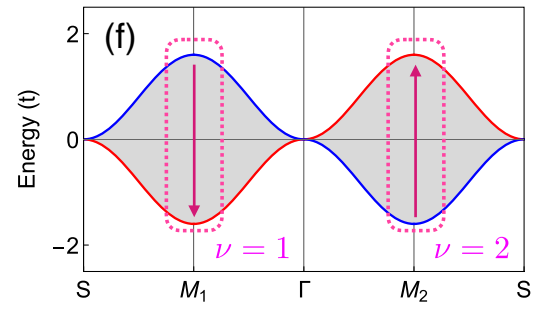

FIG. 1. Archetype GMR and TMR model mechanisms in unconventional antiferromagnets. Red and blue correspond to up and down spins, respectively, and a gray arrow marks the applied current direction. (a) The GMR stack with a metallic spacer in a current-in-plane geometry. As an example, we show the antiparallel configuration of the Néel vectors in the two electrodes $\mathrm{AF}_{1}$ and $\mathrm{AF}_{2}$. Interfaces are oriented along one of the main axes of the elliptic spin-dependent bands. (b) Energy band cuts highlighting the anisotropic alternating spin-momentum coupling around the $\boldsymbol{\Gamma}$ point in the Brillouin zone, resulting in anisotropic spin-dependent conductivities. (c) The tightbinding model band dispersion. The dashed rectangle highlights the region with the anisotropic spin-momentum coupling around the $\boldsymbol{\Gamma}$ point. (d) The TMR stack with an insulating barrier. As an example, we show the parallel configuration of the Néel vectors in the two electrodes. (e) Energy band cuts highlighting the valley-dependent alternating spin-momentum coupling around the $\mathbf{M}_{1}$ and $\mathbf{M}_{2}$ points in the Brillouin zone, resulting in valley and spin-dependent densities of states. (f) The tight-binding model band dispersion. The dashed rectangles highlight the valleys with opposite spin splitting, marked by the magenta arrows.

Research in electrical detection and manipulation of the antiferromagnetic order turned, instead, to reading and writing principles based on relativistic spin-orbit coupling phenomena [13-23]. The resulting successful demonstrations of experimental memory devices, showcasing among others the insensitivity of antiferromagnets to magnetic fields or their ultrafast dynamics, prompted extensive fundamental and applied research interest in antiferromagnetic spintronics [18,24-30]. However, the realization of antiferromagnetic counterparts of the nonrelativistic GMR, TMR, and STT phenomena, driven by robust conserved spin currents, remained elusive.

In this paper, we theoretically develop a GMR and TMR concept [31] based on unconventional collinear antiferromagnetism with a nonrelativistic alternating spinmomentum coupling of two forms illustrated in Fig. 1. The first form, which we show enables the GMR, has anisotropic spin-polarized bands resulting in different conductivities of the opposite spin channels despite the zero equilibrium magnetization [32-37] [Figs. 1(a)-1(c)]. The second form, favoring the TMR, has spin-polarized band-structure valleys with the corresponding spin-dependent densities of states [31] [Figs. 1(d)-1(f)].

Since the alternating spin-momentum coupling plays a central role in our study, we first summarize its key characteristics as emerging from recent literature [3136,38]. Next, we present our archetype GMR and TMR models with the anisotropic and valley-dependent spinmomentum couplings. We then discuss the correspondence of these models to density-functional-theory (DFT) calculations in the unconventional antiferromagnetic phases of representative materials $\mathrm{RuO}_{2}$ [32-34,36,37] and $\mathrm{Mn}_{5} \mathrm{Si}_{3}$ [31]. Based on the DFT calculations of spindependent conductivities and densities of states, we predict large magnitudes of GMR and TMR in these materials. In the final section, we discuss GMR and TMR in the unconventional collinear antiferromagnets from a broader materials perspective, focusing on the general symmetry criteria. We conclude the section by proposing that, apart from the GMR and TMR detection, the alternating spinmomentum coupling can also facilitate magnetic excitation by the STT. 


\section{NONRELATIVISTIC ALTERNATING SPIN-MOMENTUM COUPLING}

DFT and model calculations by several groups reveal lifted spin degeneracies in the nonrelativistic band structure of unconventional collinear antiferromagnetic crystals which lack a symmetry combining time reversal with space inversion or translation [31-43]. The phenomenon is microscopically associated with a Fermiliquid instability in an anisotropic spin interaction channel [33] and with anisotropic real-space magnetization densities $[32,37]$. Formalisms of electromagnetic multipoles $[34,41]$ or symmetry groups $[35,38,43,44]$ are employed to study materials exhibiting this unconventional collinear antiferromagnetism. The lifted spin degeneracy in the equilibrium band structure of the unconventional collinear antiferromagnets is linked to anomalous responses, including a Hall effect generated by the compensated collinear magnetic order $[31,32,36]$, or nonrelativistic spin currents flowing transverse to the applied electrical bias $[37,45,46]$.

The type of compensated magnetic crystal order considered in the above studies can be summarized as follows. The crystals are composed of two or multiple sublattices which can contain, besides magnetic atoms, also nonmagnetic atoms, and the corresponding elements between the sublattices are chemically equivalent. In these crystals, the nuclear-position (charge) structure has at least one spatial symmetry operation (translation, inversion, rotation, or a combination of these) that transposes one sublattice onto the other-an intersublattice transposing symmetry $[4,47]$. Nonrelativistic electron-electron Coulomb interactions can lead to quantum ground states that have nonzero magnetic moments on the sublattices, and the transposing symmetry favors states with magnetic moments of precisely equal magnitudes. An antiparallel magnetic order then can give a strictly zero net magnetization in the absence of spin-orbit coupling $[4,47]$.

The conventional spin-degenerate antiferromagnetic bands correspond to the above magnetic crystals in the case when the intersublattice transposing symmetries are inversion or translation. On the other hand, the unconventional antiferromagnetic band structures with the alternating spin-momentum coupling correspond to the case in which the transposing symmetries contain a crystal rotation operation. In the context of our GMR and TMR study, we highlight the following characteristics of the alternating spin-momentum coupling: (i) The band structure with the alternating spin-momentum coupling arises from the nonrelativistic part of the DFT Hamiltonian. (ii) It gives a zero net magnetization when integrated over the whole Brillouin zone. (iii) It enables one to define a momentum-independent spin-quantization axis. (iv) It breaks the time-reversal symmetry in the momentum space and can have lifted spin degeneracy at low-symmetry momenta, as well as at high-symmetry time-reversal-invariant momenta, except the $\boldsymbol{\Gamma}$ point. (v) The bands are inversion symmetric in the momentum space, $E_{s}(\mathbf{k})=E_{s}(-\mathbf{k})$, where $s$ is the spin index of the energy band $E_{s}(\mathbf{k})$, and, thus, are even (quadratic) in momentum. (vi) We note that the band structures with the alternating spin-momentum coupling can be classified into ten centrosymmetric spin point groups [38]. Here, in contrast to the relativistic magnetic symmetry groups, the nonrelativistic spin groups consider symmetry operations in uncoupled real and spin spaces [38,48-50] and use the decomposition into the intersublattice transposing and nontransposing symmetries $[4,31-33,38,47]$, where the latter transform a magnetic sublattice on itself. The nonrelativistic spin symmetries which determine the nonrelativistic spin-momentum coupling can be, thus, written as a pair of symmetry operations, $\left[\mathcal{C}_{2} \| \mathcal{C}_{i}\right]$, where $\mathcal{C}_{2}$ is the $180^{\circ}$ spin rotation in the spin space around an axis perpendicular to spins and $\mathcal{C}_{i}$ is the symmetry transformation in the real crystallographic space $[38,50]$. The resulting spin group can be labeled by the Hermann-Mauguin symbol of, e.g., a form $2_{m} 2_{m} 1_{m}$. Here, the normal font corresponds to the spin symmetry transformation ( 2 stands for the spin rotation and 1 for identity), while the subindex corresponds to the paired crystallographic operation ( $m$ stands for mirror) $[38,48,50]$. We use this notation for describing the nonrelativistic symmetry group of our model and of the magnetic crystals of $\mathrm{RuO}_{2}$ and $\mathrm{Mn}_{5} \mathrm{Si}_{3}$.

\section{ANISOTROPIC AND VALLEY-DEPENDENT SPIN-MOMENTUM COUPLINGS AND GMR AND TMR MODELS}

For our GMR and TMR study, it is instructive to distinguish between anisotropic and valley-dependent spin-momentum couplings, whose specific realizations are also identified in the earlier studies [31-35]. The two types of alternating spin-momentum coupling differ by their local band-structure shapes in the momentum space (see Fig. 1): The anisotropic coupling is characterized by its local band anisotropy (and does not have to exhibit opposite-polarization valleys separated in momentum). On the other hand, the valley-dependent coupling is characterized by the opposite spin-polarized valleys which are decoupled in the momentum space (and do not have to be anisotropic). We remark that we study here the two types of spin-momentum coupling in their pristine form, while, in general band structures, both types can coexist. We now describe the two types of alternating spin-momentum coupling in more detail.

The anisotropic alternating spin-momentum coupling corresponds to two perfectly spin-polarized, but mutually rotated bands which are highly anisotropic in momentum:

$$
\frac{\partial^{2} E_{s}(\boldsymbol{k})}{\partial k_{x}^{2}} \neq \frac{\partial^{2} E_{s}(\boldsymbol{k})}{\partial k_{y}^{2}}
$$


where spin is marked by $\boldsymbol{s}$ and momentum as $\boldsymbol{k}=\left(k_{x}, k_{y}\right)$. In other words, the anisotropic spin-momentum-coupled bands of a form $E_{s}(\boldsymbol{k}) \sim \alpha^{s} k_{x}^{2}+\beta^{s} k_{y}^{2}$ exhibit strongly anisotropic coefficients $\alpha^{s} \neq \beta^{s}$ (see also the explicit model Hamiltonian in the next section). We illustrate the anisotropic alternating spin-momentum coupling on the band structures in Fig. 1(b). Here, we observe the spindegenerate $\Gamma$ point accompanied by the two elliptical Fermi surfaces with opposite spin polarization. The opposite-spin Fermi surfaces are related by the $\left[\mathcal{C}_{2} \| \mathcal{C}_{4}\right]$ symmetry, which ensures that the two spin-polarized valleys are equally populated. The two spin-polarized bands, thus, exhibit spin-dependent anisotropic group velocities $\left[\partial E_{+}(\boldsymbol{k}) / \partial k_{i}\right] \neq\left[\partial E_{-}(\boldsymbol{k}) / \partial k_{i}\right]$, where \pm refers to the spin index. This results in spin-dependent anisotropic conductivities which can take the form $\sigma_{+, x x} \neq \sigma_{-, x x}$, $\sigma_{+, y y} \neq \sigma_{-, y y}$, and $\sigma_{ \pm, x x}=\sigma_{\mp, y y}$ [Fig. 1(b)]. Applying the current along, e.g., the $x$ direction, the conductivity of the GMR stack depends on whether the Néel vectors in the two layers separated by the nonmagnetic metallic spacer are parallel or antiparallel, in analogy to ferromagnetic GMR.

From the ratio of the conductivities of the spin-up and spin-down channels, $R_{\sigma}=\sigma_{+, x x} / \sigma_{-, x x}=\sigma_{+, x x} / \sigma_{+, y y}=$ $\sigma_{-, y y} / \sigma_{-, x x}$, the GMR can be estimated from the conventional current-in-plane GMR expression derived in ferromagnets [1]:

$$
\mathrm{GMR}=\frac{1}{4}\left(R_{\sigma}+\frac{1}{R_{\sigma}}-2\right) .
$$

The expression explicitly highlights that the GMR is maximized in band structures with a strong spin-dependent anisotropy. We use this expression below in the section on first-principles calculations.

The valley-dependent spin-momentum coupling is characterized by spin-polarized energy eigenstates in a region around a momentum $\mathbf{M}_{\nu}$, which typically corresponds to a band extremum:

$$
\left.\frac{\partial E_{s}(\boldsymbol{k})}{\partial \boldsymbol{k}}\right|_{\boldsymbol{k} \rightarrow \boldsymbol{M}_{\nu}}=0
$$

This is commonly referred to as a valley, and $\nu$ is the valley index. The valley-dependent spin-momentum coupling features the characteristic oppositely spin-polarized valleys, which are well separated in momentum, and where the bands can be locally isotropic, i.e., $E_{s}(\boldsymbol{k}) \sim \alpha\left(k_{x}^{2}+k_{y}^{2}\right)$, as illustrated on the circular Fermi surfaces in Fig. 1(e). These characteristics contrast the anisotropic spinmomentum coupling. The spin polarization of each momentum-space valley is perfectly compensated by a counterpart valley with the opposite spin polarization. The spin- and momentum-dependent energies in the two valleys are connected by the intersublattice transposing symmetry, containing a real-space rotation operation $\left(\mathcal{C}_{4}\right)$, augmented with a $180^{\circ}$ spin rotation marked by $\mathcal{C}_{2}$ [38], i.e., $E_{+}\left(\mathbf{M}_{1}\right)=$ $\left[\mathcal{C}_{2} \| \mathcal{C}_{4}\right] E_{-}\left(\mathbf{M}_{2}\right)$.

We emphasize that, unlike the relativistic spin-orbitcoupling-induced valleys [51], the nonrelativistic alternating spin-polarization valleys are distributed symmetrically in the momentum space $\left[E_{s}\left(\mathbf{M}_{\nu}\right)=E_{s}\left(-\mathbf{M}_{\nu}\right)\right]$, do not require inversion symmetry breaking, and can occur at time-reversal-invariant momenta [31].

The pairs of momentum-space valleys with opposite spin polarizations result in the equal net population of spin-up and spin-down states, while the densities of states within a given valley become spin dependent: $n_{+}\left(\mathbf{M}_{1}\right) \neq n_{-}\left(\mathbf{M}_{1}\right)$, $n_{+}\left(\mathbf{M}_{2}\right) \neq n_{-}\left(\mathbf{M}_{2}\right)$, and $n_{ \pm}\left(\mathbf{M}_{1}\right)=n_{\mp}\left(\mathbf{M}_{2}\right)$. For tunneling which conserves the valley index, parallel and antiparallel configurations of the Néel vectors in the two layers separated by the tunnel barrier give different conductances, in analogy to ferromagnetic TMR. We can then apply the Jullière TMR formula [1] per valley:

$$
\mathrm{TMR}=\frac{1}{2}\left(R_{n}+\frac{1}{R_{n}}-2\right),
$$

where the ratio of the spin-up and spin-down densities of states in the valley $R_{n}=n_{+}\left(\mathbf{M}_{1}\right) / n_{-}\left(\mathbf{M}_{1}\right)=$ $n_{+}\left(\mathbf{M}_{1}\right) / n_{+}\left(\mathbf{M}_{2}\right)=n_{-}\left(\mathbf{M}_{2}\right) / n_{-}\left(\mathbf{M}_{1}\right)$. Again, we use this expression below in the first-principles TMR calculations.

An important feature of the valley-dependent spinmomentum coupling is that it provides for a large momentum separation [marked by $\boldsymbol{\Delta}$ in Fig. 1(e)] of the opposite spin states at a given energy. Each of the spin-polarized momentum-space valleys corresponds to a ferromagneticZeeman-like spin-split band structure [31] and, therefore, allows for the TMR when intervalley scattering is not dominant. We remark that the alternating spin valleys shown in Fig. 1(e) are isotropic, i.e., would not generate the GMR derived from the anisotropic spin-dependent conductivities.

\section{MODEL TIGHT-BINDING HAMILTONIAN AND NUMERICAL SIMULATIONS}

We now proceed with presenting a 2D tight-binding model Hamiltonian describing the nonrelativistic alternating spin-momentum couplings. In the Hamiltonian, we parametrize the kinetic nearest-neighbor hopping by $t$ and describe the alternating spin-momentum coupling by a spin-dependent hopping parametrized by $t_{J}[31]$ :

$$
\begin{aligned}
\mathcal{H}(\mathbf{k})= & 2 t\left(\cos k_{x}+\cos k_{y}\right) \mathbf{1} \\
& +2 t_{J}\left(\cos k_{x}-\cos k_{y}\right) \boldsymbol{\sigma} \cdot \mathbf{d} .
\end{aligned}
$$

Here, $\mathbf{d}$ is a unit vector along the Néel vector, $\mathbf{1}$ is the unit matrix, and $\boldsymbol{\sigma}$ is the vector of Pauli spin matrices.

The Hamiltonian describes a magnetic lattice shown in Fig. S1 in Supplemental Material [52]. The model 
(a)

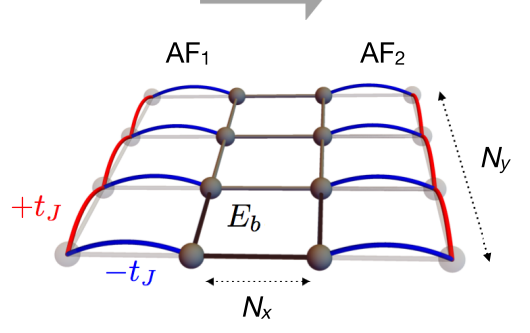

(d)

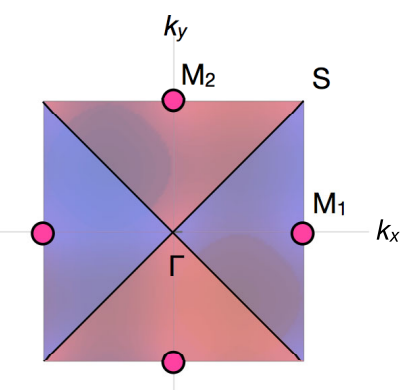

(b)

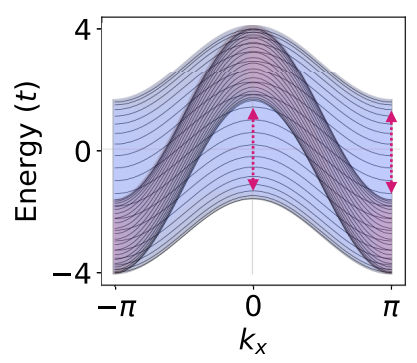

(c) $C\left(\frac{\mathrm{e}^{2}}{h}\right) \frac{C_{P}-C_{A P}}{C_{P}+C_{A P}}(\%)$

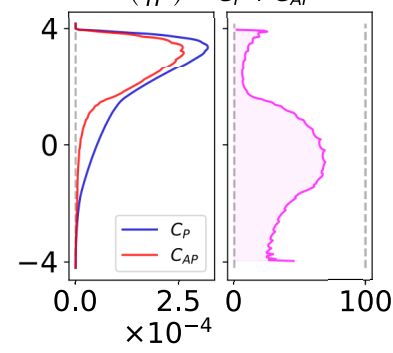

(e)
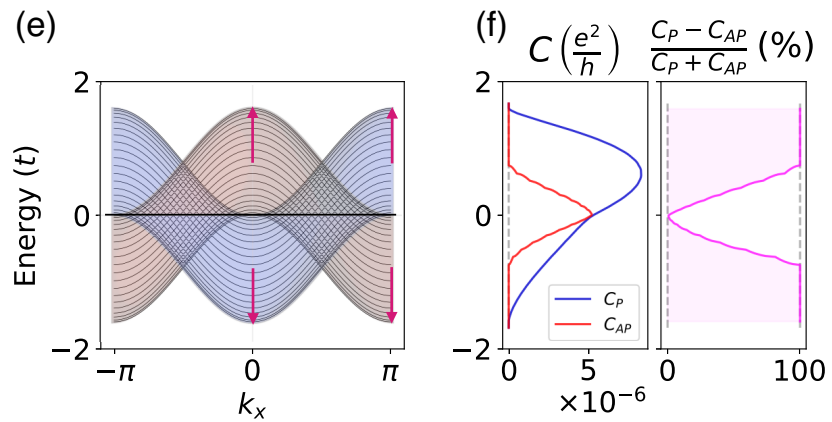

FIG. 2. Model transport calculations in a quasi-two-dimensional tunnel junction. (a) Schematics of the geometry of the leads representing the unconventional antiferromagnets, the scattering region separating the leads (spacer), and selected parameters used in the calculations. (b) Spin-up (red) and spin-down (blue) energy bands in the lead as a function of $k_{x}$ plotted for the discrete set of transverse wave-vector parameters corresponding to $N_{y}=20$ lattice points in the direction parallel to the lead-spacer interface. Hamiltonian parameters in the lead correspond to Fig. 1(c) and the first case described in the text. (c) Conductances for parallel (P) and antiparallel (AP) configurations of the Neel vectors in the leads and the corresponding relative difference of the P and AP conductances. (d) Top view of the energy bands with the alternating spin-momentum coupling in the lead in the first Brillouin zone. Hamiltonian parameters in the lead correspond to Fig. 1(f) and the second case described in the text. Magenta circles mark the alternating spin-polarized valleys. (e),(f) The same as (b),(c) for Hamiltonian parameters in the lead corresponding to Fig. 1(f) and the second case described in the text. In (b),(e), the magenta arrows mark the energy ranges with decoupled spin-up and spin-down channels due to the spin-polarized valleys in the band structure.

corresponds to a (2D) nonrelativistic spin symmetry space (point) group $P 2{ }_{4} 1_{m} 2_{m}\left(2{ }_{4} 1_{m} 2_{m}\right)$, which lacks inversion and translation intersublattice transposing symmetries but exhibits a transposing symmetry containing a real-space rotation. (We use the Hermann-Mauguin notation of the nonrelativistic spin symmetry groups $[38,48,50]$.)

The spin-up (+) and spin-down (-) energy bands are given by

$E_{ \pm}(\mathbf{k})=2 t\left(\cos k_{x}+\cos k_{y}\right) \pm 2 t_{J}\left(\cos k_{x}-\cos k_{y}\right)$.

The anisotropic spin-momentum coupling, shown in Fig. 1(b) and highlighted by a dashed rectangle in Fig. 1(c), is obtained by performing the $\mathbf{k} \cdot \mathbf{p}$ approximation around the $\boldsymbol{\Gamma}$ point, for which the energy spectrum can be written as

$$
E_{ \pm}(\mathbf{k})=4 t-\left(t \pm t_{J}\right) k_{x}^{2}-\left(t \mp t_{J}\right) k_{y}^{2},
$$

and by taking, e.g., $t>0$ and $t_{J}=0.4 t$.
On the other hand, the valley-dependent spin-momentum coupling shown in Fig. 1(e) and highlighted by dashed rectangles in Fig. 1(f) is obtained from Eq. (6) by performing the $\mathbf{k} \cdot \mathbf{p}$ approximation in $\mathbf{M}_{1}$ and $\mathbf{M}_{2}$ valleys. For $t=0$ and $t_{J}>0$, we obtain

$$
\begin{aligned}
& E_{ \pm}\left(\mathbf{M}_{1}, \mathbf{k}\right)= \pm t_{J}\left(4-k^{2}\right) \\
& E_{ \pm}\left(\mathbf{M}_{2}, \mathbf{k}\right)=\mp t_{J}\left(4-k^{2}\right) .
\end{aligned}
$$

Before discussing the DFT calculations, we show in Fig. 2 numerical simulations of the quantum conductance of a tunnel junction based on the tight-binding model (5). We consider two illustrative sets of parameters. The first case, shown in Figs. 2(b) and 2(c), has the Hamiltonian parameters in the leads corresponding to Fig. 1(c), i.e., $t=$ $t^{L}>0$ and $t_{J}=t_{J}^{L}=0.4 t^{L}$. The second case, shown in Figs. 2(d)-2(f), corresponds ro the Hamiltonian parameters of Fig. 1(f), i.e., $t=t^{L}=0$ and $t_{J}=t_{J}^{L}>0$. In both cases, we model the nonmagnetic tunneling barrier by setting the Hamiltonian parameters in the barrier to $t_{J}=t_{J}^{B}=0$ and 
$t=t^{B}=0.025 t_{J}^{L}$. In the barrier, we also add an on-site energy term $E_{B}=10 t_{J}^{L}$. We set the number of sites in the barrier along the $x$ axis to $N_{x}=2$ and in the barrier and leads along the $y$ axis to $N_{y}=20$ [Fig. 2(a)].

The transport calculations are done using the Kwant package [53]. (For more details on our three-dimensional antiferromagnetic junctions, see Supplemental Material [52], Secs. 1 and 2). As anticipated in the above discussion of Fig. 1, we obtain a higher conductance for the parallel than for the antiparallel Néel vector configurations [Figs. 2(c) and 2(f)].

The sensitivity of the ratio $R=\left[\left(C_{\mathrm{P}}-C_{\mathrm{AP}}\right) /\left(C_{\mathrm{P}}+C_{\mathrm{AP}}\right)\right]$ to the transport energy and model parameters can be understood by analyzing the spin-projected energy bands of the leads shown in Figs. 2(b) and 2(e). According to our qualitative analysis in Fig. 1, we expect the limiting ratio $R \approx 100 \%$ for the energy regions of the band structure where the spin-up and spin-down channels are decoupled and well separated in the momentum space. This situation arises for the second set of Hamiltonian parameters for the energy regions marked by the magenta arrows in Fig. 2(e), i.e., for $E \gtrsim 0.8 t$ and $E \lesssim-0.8 t$. The energy states in a given valley exhibit perfect spin polarization of one sign and are well separated in the momentum space from the valley containing the opposite spin states [see $\Delta$ in Fig. 1(e)]. As a result, the opposites spins are decoupled for all transport $k_{x}$ channels, and the tunneling sensitivity to the mutual orientation of the Néel vectors in the two electrodes is strongly enhanced, resulting in $R \approx 100 \%$ in Fig. 2(f). In contrast, for zero energy, the spin-up and spin-down bands are degenerate and form the nodal lines [31] marked by the black lines in Figs. 2(d) and 2(e). This explains the suppression of $R$ around zero energy, seen in Fig. 2(f).

For the first set of Hamiltonian parameters, the situation is different. Within the energy bands, there is no energy region where the spin-up and spin-down transport channels are completely decoupled; i.e., the spin-up and spin-down bands partly overlap [see Fig. 2(b)]. However, the $\mathbf{M}_{1(2)}$ valley splittings are still present, marked by magenta arrows in Fig. 2(b). The corresponding energy regions exhibit the largest conductance ratio $R$ [see Fig. 2(c) around zero energy]. We note that the particle-hole asymmetry in the conductance signal arises as the states with higher energies, i.e., closer to the top of the tunneling barrier, have a higher tunneling probability.

In summary, a large tunneling magnetoresistance is achieved at the energy regions corresponding to the alternating spin-polarized valleys. The model tunnel-junction simulations, thus, corroborate our qualitative analysis in Fig. 1.

\section{FIRST-PRINCIPLES CALCULATIONS IN $\mathrm{RuO}_{2}$ AND $\mathrm{Mn}_{5} \mathrm{Si}_{3}$}

We start our discussion of first-principles GMR and TMR calculations with $\mathrm{RuO}_{2}$, which crystallizes in a rutile structural space group $P 4_{2} / \mathrm{mnm}$. The unconventional antiferromagnetic order shown in Fig. 3(a) corresponds to the nonrelativistic spin space (point) group $P 2_{4_{2}} 1_{/ m} 2_{n} 1_{m}\left(2_{4} 1_{/ m} 2_{m} 1_{m}\right)$ [38]. The spin group lacks inversion and translation intersublattice transposing symmetries but exhibits a transposing symmetry containing a real-space rotation $\mathcal{C}_{4 z}$ [see Fig. 3(a)] [38]. As a result, $\mathrm{RuO}_{2}$ has the band structure with the nonrelativistic alternating spin-momentum coupling $[32,33,37,38]$, analogous to the 2D tight-binding model discussed in the previous section.

We calculate the electronic structure in the pseudopotential DFT code Vienna Ab Initio Simulation Package (VASP) [54], within Perdew-Burke-Ernzerhof (PBE) $+U+$ SOC (a spherically invariant version of DFT $+U$ ), and we use an energy cutoff of $500 \mathrm{eV}$. We set the Hubbard $U$ to $1.6 \mathrm{eV}$. The lattice parameters are $a=b=4.5331$ and $c=$ $3.1241 \AA$ [32]. Wannier functions are obtained using the Wannier90 code [55]. The longitudinal conductivity is calculated separately for each spin channel using the Boltzmann equation with a $160^{3}$ crystal momentum mesh and with the scattering rate of $6.6 \mathrm{meV}$ corresponding to the experimental conductivity [36]. (We also confirm the results by calculations using the WannierBerri code [56], which we show in Supplemental Material [52].)

The spin-resolved band structure is shown in Figs. 3(b) and 3(c), and the spin-dependent conductivities and GMR are plotted in Figs. 3(d) and 3(e). The dashed rectangles in Fig. 3(c) depict the parts of the spectrum with the anisotropic spin-momentum coupling, corresponding to our GMR model in Figs. 1(a)-1(c). The anisotropic spin-dependent group velocities in this part of the spectrum are further highlighted in Fig. 3(b). As expected from our model discussion, the difference between the conductivities of the opposite spin channels, and the corresponding GMR magnitude, are maximized when the Fermi level crosses the regions with the anisotropic spin-momentum coupling. Here, the GMR reaches a $100 \%$ scale. We note the correspondence of our GMR with the earlier evaluated transverse spin currents in $\mathrm{RuO}_{2}$, which also originate from the difference between the spin-up and spin-down conductivities [37].

Figure 4(a) shows DFT calculations of momentumand sublattice-resolved energy bands in $\mathrm{RuO}_{2}$, while Fig. 4(b) shows sublattice- and spin-resolved densities of states. When combined, these two figures can be used to estimate the TMR in $\mathrm{RuO}_{2}$. For example, when focusing on energies around $0.5 \mathrm{eV}$ [see the dotted line in Figs. 4(a) and 4(b)], we see from Fig. 4(b) that spin-up electrons are primarily on the spin sublattice "1." In Fig. 4(a), we then see a valley between $\boldsymbol{\Gamma}$ and $\mathbf{S}$ points associated with the sublattice 1 . This valley has a spin-up polarization. On the other hand, the valley between $\boldsymbol{\Gamma}$ and $\mathbf{S}^{\prime}$ points has a spin-down polarization. In this part of the spectrum, the unconventional antiferromagnet effectively acts as two 
(a)

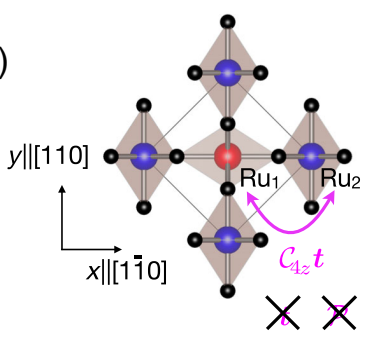

(b)

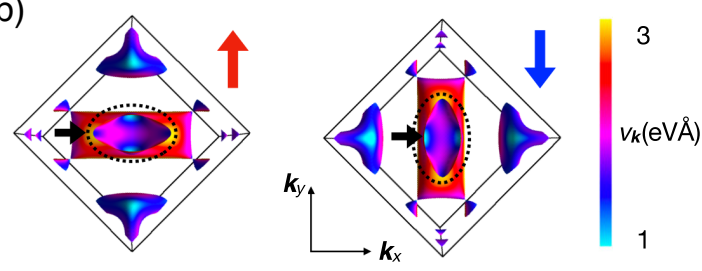

(c) $\quad(\pi,-\pi, 0) \quad(0,0,0) \quad(\pi, \pi, 0) \quad(\pi, \pi, \pi) \quad(0,0, \pi)$

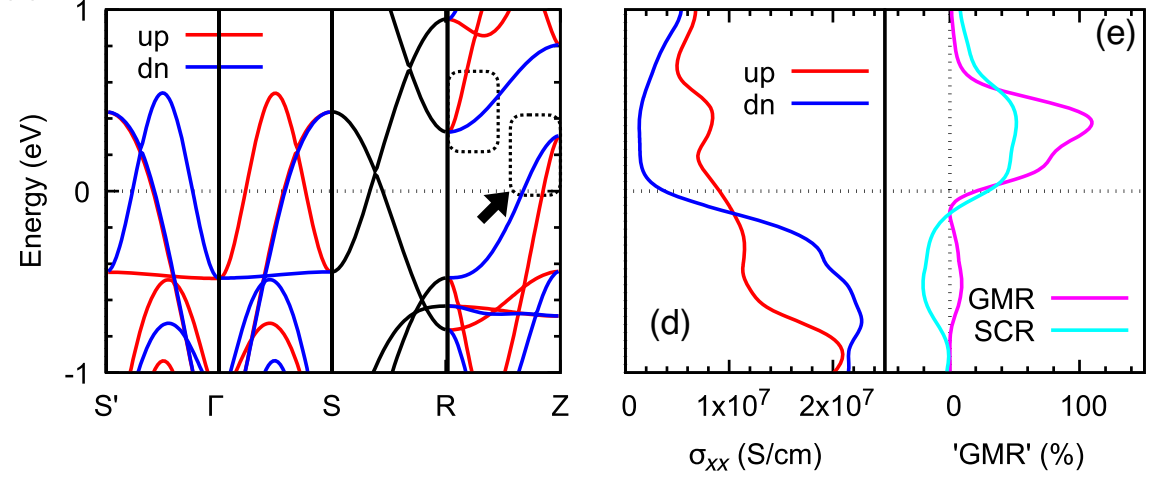

FIG. 3. First-principles calculations of GMR in $\mathrm{RuO}_{2}$ [32]. (a) Crystal structure of $\mathrm{RuO}_{2}$. Red and blue mark opposite magnetic moments on the first and second Ru sublattices. The presence of an intersublattice transposing symmetry containing a crystal rotation operation $\left(\mathcal{C}_{4 z}\right)$ and the absence of translation $(t)$ and inversion $(P)$ transposing symmetries are also highlighted. (b) Fermi surface plots for spin-up (left) and spin-down (right) states. The color coding corresponds to the group velocities, highlighting the strong anisotropy of the two spin-channel conductivities. (c) Spin-projected nonrelativistic energy bands of $\mathrm{RuO}_{2}$ with marked anisotropic spin-momentum coupling around $\mathbf{R}$ and $\mathbf{Z}$ points. (d) Longitudinal spin-up and spin-down conductivities. (e) GMR ratio. For comparison, also shown is the transverse spin current relative to the longitudinal charge current (SCR) calculated in Ref. [37].

intertwined half-metallic ferromagnets. For a valley-conserved tunneling, the TMR can be then estimated from the ratio of spin-up and spin-down densities of states projected on a sublattice. We emphasize that this approach is applicable only when the band structure has the valleydependent form of the alternating spin-momentum coupling. In agreement with our model discussions in Figs. 1 and 2, the DFT TMR ratio is maximized at energies with (a)
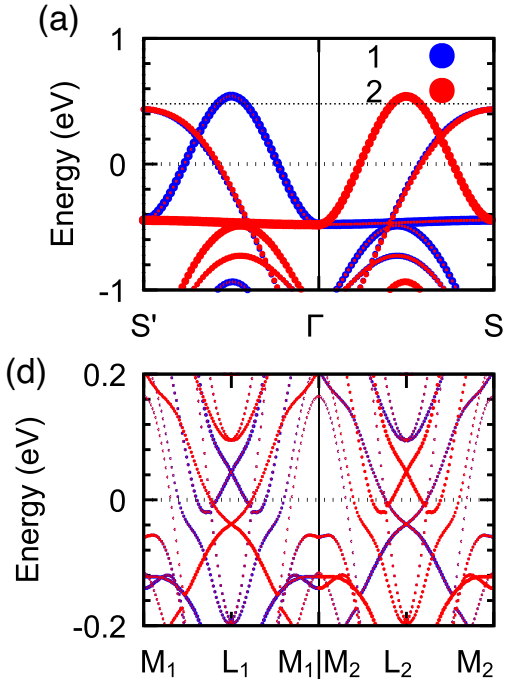

(b)
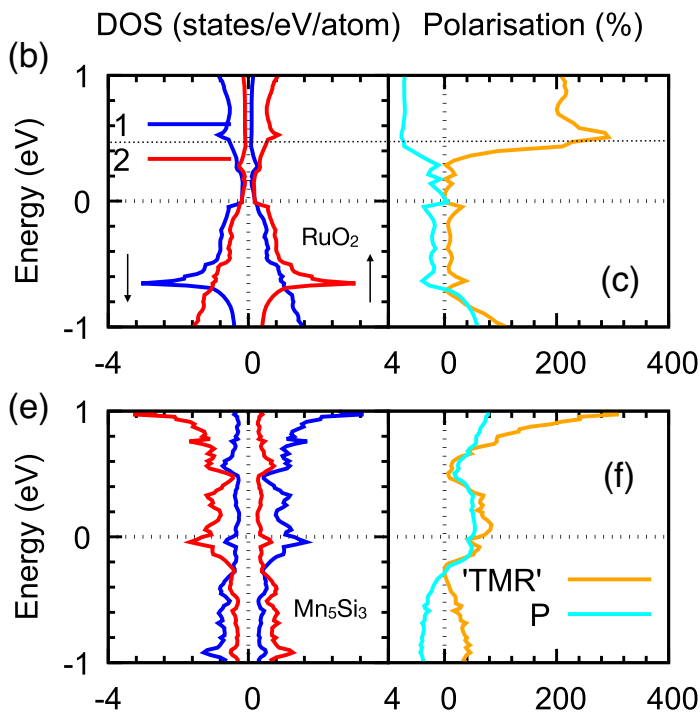

FIG. 4. First-principles calculations of $\mathrm{TMR}$ in $\mathrm{RuO}_{2}$ [32] and $\mathrm{Mn}_{5} \mathrm{Si}_{3}$ [31]. (a)-(c) are for $\mathrm{RuO}_{2}$ and (d)-(f) for $\mathrm{Mn}_{5} \mathrm{Si}_{3}$. (a) $\mathrm{Wave}$ vector- and sublattice- (blue and red, respectively) resolved energy bands. (b) Sublattice- (blue and red) and spin- (left and right) resolved density of states as a function of energy. (c) Energy-dependent TMR and spin polarization parameter (see the text). (d)-(f) The same as (a)-(c) for $\mathrm{Mn}_{5} \mathrm{Si}_{3}$. 
the dominant contribution from the spin-polarized valleys, where it reaches a $100 \%$ scale.

On a quantitative level, $\mathrm{RuO}_{2}$ is not optimal for TMR because of the low and weakly spin-dependent density of states at the Fermi level. A more favorable candidate is $\mathrm{Mn}_{5} \mathrm{Si}_{3}$. It has a structural space group $P 6_{3} / \mathrm{mcm}$. The four-sublattice checkerboard-antiparallel magnetic order [31], generated by the nonrelativistic electron-electron Coulomb interactions, is again precisely compensated, owing to the presence of the intersublattice transposing symmetry. Its nonrelativistic spin space (point) group $P 1_{m} 2_{m} 2_{a}\left(1_{m} 2_{m} 2_{m}\right)$ lacks inversion and translation transposing symmetries $[31,38]$. Correspondingly, it also has the band structure with the alternating spin-momentum coupling. Unlike $\mathrm{RuO}_{2}$, however, it contains spin-polarized valleys at time-reversal-invariant momenta $\mathbf{M}_{1}$ and $\mathbf{M}_{2}$ [31], analogous to the model in Figs. 1(d)-1(f). Additional spin-polarized channels are found at the $\mathbf{L}_{1}$ and $\mathbf{L}_{2}$ points of the $\mathrm{Mn}_{5} \mathrm{Si}_{3}$ Brillouin zone. Our DFT calculations plotted in Figs. 4(d)-4(f) show that this metallic antiferromagnet with the valley-dependent spin-momentum coupling at high-symmetry points in the Brillouin zone is a favorable candidate for large TMR ratios even at the Fermi level.

For illustration, we plot in Figs. 4(c) and 4(f) also the spin-polarization parameter given by the relative difference of the sublattice-projected spin-up and spin-down densities of states. We see that its energy dependence correlates with the dependence of the TMR ratio.

\section{DISCUSSION}

We now discuss the GMR and TMR in unconventional antiferromagnets from a more general materials perspective. Apart from $\mathrm{RuO}_{2}$ and $\mathrm{Mn}_{5} \mathrm{Si}_{3}$, other materials were previously identified to host nonrelativistic alternating spin-momentum coupling, including $\mathrm{MnF}_{2}$ [35], $\mathrm{CaCrO}_{3}$ [46], or organic crystals [45]. However, these may not be optimal materials for GMR and TMR, because they either are insulating $[35,45]$ or exhibit only a weakly anisotropic spin-momentum coupling [46]. Additional candidates can be found in databases of magnetic materials that were recently discussed from the perspective of the nonrelativistic spin-split band structures in unconventional antiferromagnets $[41,43]$. However, only structural and relativistic magnetic symmetry groups of the materials are listed. The determination of the nonrelativistic spin symmetry groups for the extensive set of materials in the magnetic databases, thus, represents an important future task.

To connect the nonrelativistic spin symmetry group classification of the band structures [38] to GMR and TMR, we now discuss the nonrelativistic symmetry criteria for the two effects in the unconventional antiferromagnets. In principle, TMR can be realized in all materials with nonrelativistic alternating spin-momentum coupling. Their band structures can then be associated with one of the ten centrosymmetric spin point groups listed in Ref. [38].
In addition, we explicitly show in the present work that TMR is enhanced in systems with valley-dependent spinmomentum coupling. Here, the spin-polarized valleys can form around high-symmetry points in the Brillouin zone, such as the $\mathbf{M}_{1,2}$ valleys in $\mathrm{Mn}_{5} \mathrm{Si}_{3}$, or around lowsymmetry points, such as the band extrema along the $\boldsymbol{\Gamma}-\mathbf{S}$ lines in $\mathrm{RuO}_{2}$. However, an enhanced TMR can arise also from more generic spin-polarized quasiparticles outside the band extrema, as long as they are well separated in the momentum space and provide for the sufficiently decoupled spin transport channels. (A systematic analysis of the general spin-polarized quasiparticles favorable for a large TMR in unconventional antiferromagnets is beyond the scope of the present work.)

The current-in-plane GMR, derived from the anisotropic spin-momentum coupling and the corresponding anisotropic spin-dependent conductivities, is symmetrywise more restrictive. We find that only four out of the ten centrosymmetric spin point groups classifying the band structures with the alternating spin-momentum coupling [38] allow for GMR. Specifically, these four GMR spin point groups are $22_{2} 2_{m}, 2{ }_{m} 2_{m} 1_{m}, 2{ }_{4} 1_{/ m}$, and $2_{4} 1_{/ m} 2_{m} 1_{m}$.

In terms of robustness, we point out that the nonrelativistic symmetry criteria for GMR and TMR do not depend on the orientation of the Néel vector with respect to the crystal axes and that the alternating spin-momentum coupling is due to the nonrelativistic part of the DFT Hamiltonian. The weaker relativistic spin-orbit coupling then typically plays only a minor role (see Supplemental Material [52] and Ref. [37]).

The spin polarization in the transport channels can decohere due to, e.g., electron-electron or disorder scattering. However, calculations confirm the persistence of alternating spin-momentum coupling even in the presence of strong electronic correlations [33]. In analogy to conventional valleytronics in 2D materials [51], the opposite spin polarization also suppresses intervalley scattering in unconventional antiferromagnets. In addition, we recall that the typically dominant small-angle elastic scattering off isotropic impurities does not significantly mix states from different valleys, as long as they are well separated in momentum space.

Next, we comment on the comparison from the symmetry perspective of the GMR and TMR and the anomalous Hall effect (AHE), which is another transport tool to detect the Néel vector reversal. Alternating spinmomentum coupling is shown to provide the timereversal symmetry-breaking mechanism for the AHE in the unconventional collinear antiferromagnets $\mathrm{RuO}_{2}$ or $\mathrm{Mn}_{5} \mathrm{Si}_{3}[31,32,36,57]$. However, in case of the AHE, the time-reversal symmetry breaking is connected to the transverse electrical response by the relativistic spin-orbit coupling $[31,32,36,57]$. The symmetry criteria for the AHE are, therefore, fundamentally distinct from those of 
TMR or GMR. They are formulated by means of the relativistic magnetic symmetry groups with coupled symmetry operations in the real and spin space. The symmetry criterion is that the relativistic magnetic group allows for the presence of an odd-under-time-reversal axial vector $[32,57]$. Note that both the Hall effect and magnetization follow this symmetry criterion, and, indeed, the presence of the Hall effect in the unconventional antiferromagnets can be accompanied by the formation of a weak relativistic magnetization $[31,32,36,57]$.

All the anomalous Hall antiferromagnets can be associated with one of the ten relativistic centrosymmetric magnetic point groups which fulfill the above symmetry criterion [57]. We emphasize that these are unrelated to the ten nonrelativistic centrosymmetric spin point groups classifying the band structures with alternating spinmomentum coupling [38]. For instance, the nonrelativistic spin symmetry group of $\mathrm{RuO}_{2}$, which enables GMR and TMR, is independent of the orientation of the Néel vector. On the other hand, the relativistic magnetic symmetry group of $\mathrm{RuO}_{2}$ changes when the Néel vector is rotated from the [001] axis to the (001) plane, and only in the latter case is the AHE allowed by symmetry $[32,36,57]$.

In the final paragraphs, we comment on our anisotropic and valley-dependent spin-momentum couplings in the context of the excitation by the STT. In ferromagnetic stacks, in the limit of long carrier spin lifetime, injected carriers with spin polarization $\mathbf{p}$ from one ferromagnet precess around the magnetization $\mathbf{m}$ of the other ferromagnet. The resulting nonequilibrium spin polarization in the second ferromagnet, $\mathbf{s} \sim \mathbf{m} \times \mathbf{p}$, depends on the magnetization $\mathbf{m}$. The corresponding (anti)dampinglike STT, $\mathbf{T} \sim \mathbf{m} \times(\mathbf{m} \times \mathbf{p})$, can compete with the Gilbert damping and, thus, excite the second ferromagnet [2].

Earlier studies have already demonstrated that antiferromagnets, including conventional ones with spin-degenerate bands, can be also efficiently excited by a spin-polarized current injected into the antiferromagnet $[16,18,58]$. Here, local nonequilibrium spin polarizations driving the (anti) dampinglike STT at sublattice " 1 " and " 2 ," $\mathbf{s}_{1} \sim \mathbf{m}_{1} \times \mathbf{p}$ and $\mathbf{s}_{2} \sim \mathbf{m}_{2} \times \mathbf{p}$, have opposite sign on the two sublattices since $\mathbf{m}_{1}=-\mathbf{m}_{2}$. This makes the (anti)dampinglike STT due to the injected spin current principally equally efficient in antiferromagnets as in ferromagnets $[16,18,58]$.

Previously, the considered spin-current injectors into the antiferromagnet were either ferromagnetic or relativistic $[16,18,58]$. Our present study implies that nonrelativistic alternating spin-momentum coupling allows for spincurrent injection also from an unconventional antiferromagnet. As in the case of GMR and TMR, anisotropic spin-momentum coupling is more favorable for STT in metallic stacks and in the current-in-plane geometry, while valley-dependent spin-momentum coupling is more suitable for STT in the tunneling structures.

\section{ACKNOWLEDGMENTS}

We acknowledge funding from the Czech Science Foundation Grants No. 19-18623X and No. 21-28876J, the Ministry of Education of the Czech Republic Grant No. LM2018110, LNSM-LNSpin, the EU FET Open RIA Grant No. 766566, Deutsche Forschungsgemeinschaft Grant No. TRR 173268565370 (Project No. A03), and Johannes Gutenberg University Grant TopDyn, the computing time granted on the supercomputer Mogon at Johannes Gutenberg University Mainz.

Note added.-Recently, we became aware of a related study by Shao et al. [59] on TMR in an antiferromagnetic tunnel junction.

[1] C. Chappert, A. Fert, and F. N. Van Dau, The Emergence of Spin Electronics in Data Storage, Nat. Mater. 6, 813 (2007).

[2] D. C. Ralph and M. D. Stiles, Spin Transfer Torques, J. Magn. Magn. Mater. 320, 1190 (2008).

[3] A. Brataas, A. D. Kent, and H. Ohno, Current-Induced Torques in Magnetic Materials, Nat. Mater. 11, 372 (2012).

[4] E. A. Turov, Physical Properties of Magnetically Ordered Crystals (Academic, New York, 1965).

[5] L. Néel, Magnetism and Local Molecular Field, Science 174, 985 (1971).

[6] A. S. Núñez, R. A. Duine, P. Haney, and A. H. MacDonald, Theory of Spin Torques and Giant Magnetoresistance in Antiferromagnetic Metals, Phys. Rev. B 73, 214426 (2006).

[7] C. Sürgers, G. Fischer, P. Winkel, and H. V. Löhneysen, Large Topological Hall Effect in the Non-collinear Phase of an Antiferromagnet, Nat. Commun. 5, 3400 (2014).

[8] C. Sürgers, W. Kittler, T. Wolf, and H. V. Löhneysen, Anomalous Hall Effect in the Noncollinear Antiferromagnet Mn5Si3, AIP Adv. 6, 055604 (2016).

[9] N. J. Ghimire, A. S. Botana, J. S. Jiang, J. Zhang, Y.-S. Chen, and J. F. Mitchell, Large Anomalous Hall Effect in the Chiral-Lattice Antiferromagnet CoNb3S6, Nat. Commun. 9, 3280 (2018).

[10] L. Šmejkal, J. Železný, J. Sinova, and T. Jungwirth, Electric Control of Dirac Quasiparticles by Spin-Orbit Torque in an Antiferromagnet, Phys. Rev. Lett. 118, 106402 (2017).

[11] A. H. MacDonald and M. Tsoi, Antiferromagnetic Metal Spintronics, Phil. Trans. R. Soc. A 369, 3098 (2011).

[12] H. B. M. Saidaoui, A. Manchon, and X. Waintal, Spin Transfer Torque in Antiferromagnetic Spin Valves: From Clean to Disordered Regimes, Phys. Rev. B 89, 174430 (2014).

[13] A. B. Shick, S. Khmelevskyi, O. N. Mryasov, J. Wunderlich, and T. Jungwirth, Spin-Orbit Coupling Induced Anisotropy Effects in Bimetallic Antiferromagnets: A Route towards Antiferromagnetic Spintronics, Phys. Rev. B 81, 212409 (2010).

[14] B. G. Park, J. Wunderlich, X. Martí, V. Holý, Y. Kurosaki, M. Yamada, H. Yamamoto, A. Nishide, J. Hayakawa, H. Takahashi, A. B. Shick, and T. Jungwirth, A Spin-Valve-like 
Magnetoresistance of an Antiferromagnet-Based Tunnel Junction, Nat. Mater. 10, 347 (2011).

[15] X. Marti, I. Fina, C. Frontera, J. Liu, P. Wadley, Q. He, R. J. Paull, J. D. Clarkson, J. Kudrnovský, I. Turek, J. Kuneš, D. Yi, J.-H. Chu, C. T. Nelson, L. You, E. Arenholz, S. Salahuddin, J. Fontcuberta, T. Jungwirth, and R. Ramesh, Room-Temperature Antiferromagnetic Memory Resistor, Nat. Mater. 13, 367 (2014).

[16] J. Železný, H. Gao, K. Výborný, J. Zemen, J. Mašek, A. Manchon, J. Wunderlich, J. Sinova, and T. Jungwirth, Relativistic Néel-Order Fields Induced by Electrical Current in Antiferromagnets, Phys. Rev. Lett. 113, 157201 (2014).

[17] P. Wadley, B. Howells, J. Železný, C. Andrews, V. Hills, R. P. Campion, V. Novák, K. Olejník, F. Maccherozzi, S. S. Dhesi, S. Y. Martin, T. Wagner, J. Wunderlich, F. Freimuth, Y. Mokrousov, J. Kuneš, J. S. Chauhan, M. J. Grzybowski, A. W. Rushforth, Kw. Edmond, B. L. Gallagher, and T. Jungwirth, Electrical Switching of an Antiferromagnet, Science 351, 587 (2016).

[18] T. Jungwirth, X. Marti, P. Wadley, and J. Wunderlich, Antiferromagnetic Spintronics, Nat. Nanotechnol. 11, 231 (2016).

[19] P. Wadley, S. Reimers, M. J. Grzybowski, C. Andrews, M. Wang, J. S. Chauhan, B. L. Gallagher, R. P. Campion, K. W. Edmonds, S.S. Dhesi, F. Maccherozzi, V. Novak, J. Wunderlich, and T. Jungwirth, Current Polarity-Dependent Manipulation of Antiferromagnetic Domains, Nat. Nanotechnol. 13, 362 (2018).

[20] H. Chen, Q. Niu, and A. H. MacDonald, Anomalous Hall Effect Arising from Noncollinear Antiferromagnetism, Phys. Rev. Lett. 112, 017205 (2014).

[21] J. Kübler and C. Felser, Non-collinear Antiferromagnets and the Anomalous Hall Effect, Europhys. Lett. 108, 67001 (2014).

[22] S. Nakatsuji, N. Kiyohara, and T. Higo, Large Anomalous Hall Effect in a Non-collinear Antiferromagnet at Room Temperature, Nature (London) 527, 212 (2015).

[23] A. K. Nayak, J. E. Fischer, Y. Sun, B. Yan, J. Karel, A. C. Komarek, C. Shekhar, N. Kumar, W. Schnelle, J. Kübler, C. Felser, and S. S. P. Parkin, Large Anomalous Hall Effect Driven by a Nonvanishing Berry Curvature in the Noncolinear Antiferromagnet Mn3Ge, Sci. Adv. 2, e1501870 (2016).

[24] J. Železný, P. Wadley, K. Olejník, A. Hoffmann, and H. Ohno, Spin Transport and Spin Torque in Antiferromagnetic Devices, Nat. Phys. 14, 220 (2018).

[25] P. Němec, M. Fiebig, T. Kampfrath, and A. V. Kimel, Antiferromagnetic Opto-spintronics, Nat. Phys. 14, 229 (2018).

[26] O. Gomonay, V. Baltz, A. Brataas, and Y. Tserkovnyak, Antiferromagnetic Spin Textures and Dynamics, Nat. Phys. 14, 213 (2018).

[27] L. Šmejkal, Y. Mokrousov, B. Yan, and A. H. MacDonald, Topological Antiferromagnetic Spintronics, Nat. Phys. 14, 242 (2018).

[28] V. Baltz, A. Manchon, M. Tsoi, T. Moriyama, T. Ono, and Y. Tserkovnyak, Antiferromagnetic Spintronics, Rev. Mod. Phys. 90, 015005 (2018).
[29] C. Song, Y. You, X. Chen, X. Zhou, Y. Wang, and F. Pan, How to Manipulate Magnetic States of Antiferromagnets, Nanotechnology 29, 112001 (2018).

[30] S. A. Siddiqui, J. Sklenar, K. Kang, M. J. Gilbert, A. Schleife, N. Mason, and A. Hoffmann, Perspective on Metallic Antiferromagnets, J. Appl. Phys. 128, 040904 (2020).

[31] H. Reichlova, R. L. Seeger, R. González-Hernández, I. Kounta, R. Schlitz, D. Kriegner, P. Ritzinger, M. Lammel, M. Leiviskä, V. Petiček, P. Doležal, E. Schmoranzerova, A. Bad, A. Thomas, V. Baltz, L. Michez, J. Sinova, S. T. B. Goennenwein, T. Jungwirth, and L. Smejkal, Macroscopic Time Reversal Symmetry Breaking Arising from Antiferromagnetic Zeeman Effect, arXiv:2012.15651v2.

[32] L. Šmejkal, R. González-Hernández, T. Jungwirth, and J. Sinova, Crystal Time-Reversal Symmetry Breaking and Spontaneous Hall Effect in Collinear Antiferromagnets, Sci. Adv. 6, eaaz8809 (2020).

[33] K.-H. Ahn, A. Hariki, K.-W. Lee, and J. Kuneš, Antiferromagnetism in RuO2 as d-Wave Pomeranchuk Instability, Phys. Rev. B 99, 184432 (2019).

[34] S. Hayami, Y. Yanagi, and H. Kusunose, MomentumDependent Spin Splitting by Collinear Antiferromagnetic Ordering, J. Phys. Soc. Jpn. 88, 123702 (2019).

[35] L.-D. Yuan, Z. Wang, J.-W. Luo, E. I. Rashba, and A. Zunger, Giant Momentum-Dependent Spin Splitting in Centrosymmetric Low-Z Antiferromagnets, Phys. Rev. B 102, 014422 (2020).

[36] Z. Feng, X. Zhou, L. Šmejkal, L. Wu, Z. Zhu, H. Guo, R. González-Hernández, X. Wang, H. Yan, P. Qin, X. Zhang, H. Wu, H. Chen, C. Jiang, M. Coey, J. Sinova, T. Jungwirth, and Z. Liu, Observation of the Crystal Hall Effect in a Collinear Antiferromagnet, arXiv:2002.08712.

[37] R. González-Hernández, L. Šmejkal, K. Výborný, Y. Yahagi, J. Sinova, T. Jungwirth, and J. Železný, Efficient Electrical Spin Splitter Based on Nonrelativistic Collinear Antiferromagnetism, Phys. Rev. Lett. 126, 127701 (2021).

[38] L. Šmejkal, J. Sinova, and T. Jungwirth, Altermagnetism: A Third Magnetic Class Delimited by Spin Symmetry Groups, arXiv:2105.05820.

[39] S. López-Moreno, A. H. Romero, J. Mejía-López, A. Muñoz, and I. V. Roshchin, First-Principles Study of Electronic, Vibrational, Elastic, and Magnetic Properties of FeF2 as a Function of Pressure, Phys. Rev. B 85, 134110 (2012).

[40] Y. Noda, K. Ohno, and S. Nakamura, Momentum-Dependent Band Spin Splitting in Semiconducting MnO 2: A Density Functional Calculation, Phys. Chem. Chem. Phys. 18, 13294 (2016).

[41] S. Hayami, Y. Yanagi, and H. Kusunose, Bottom-Up Design of Spin-Split and Reshaped Electronic Band Structures in Antiferromagnets without Spin-Orbit Coupling: Procedure on the Basis of Augmented Multipoles, Phys. Rev. B 102 , 144441 (2020).

[42] S. A. Egorov and R. A. Evarestov, Colossal Spin Splitting in the Monolayer of the Collinear Antiferromagnet MnF 2, J. Phys. Chem. Lett. 12, 2363 (2021).

[43] L.-D. Yuan, Z. Wang, J.-W. Luo, and A. Zunger, Prediction of Low-Z Collinear and Noncollinear Antiferromagnetic 
Compounds Having Momentum-Dependent Spin Splitting Even without Spin-Orbit Coupling, Phys. Rev. Mater. 5, 014409 (2021).

[44] S. A. Egorov, D. B. Litvin, and R. A. Evarestov, Antiferromagnetism-Induced Spin Splitting in Systems Described by Magnetic Layer Groups, J. Phys. Chem. C 125, 16147 (2021).

[45] M. Naka, S. Hayami, H. Kusunose, Y. Yanagi, Y. Motome, and H. Seo, Spin Current Generation in Organic Antiferromagnets, Nat. Commun. 10, 4305 (2019).

[46] M. Naka, Y. Motome, and H. Seo, Perovskite as a Spin Current Generator, Phys. Rev. B 103, 125114 (2021).

[47] L. D. Landau and E. M. Lifshitz, Electrodynamics of Continuous Media Course of Theoretical Physics (Pergamon, Oxford, 1984).

[48] C. Bradley and A. Cracknell, The Mathematical Theory of Symmetry in Solids: Representation Theory for Point Groups and Space Groups (Oxford University, New York, 2010).

[49] W. F. Brinkman and R. J. Elliott, Theory of Spin-Space Groups, Proc. R. Soc. A 294, 343 (1966).

[50] D. B. Litvin, Spin Point Groups, Acta Crystallogr. Sect. A 33, 279 (1977).

[51] J. R. Schaibley, H. Yu, G. Clark, P. Rivera, J. S. Ross, K. L. Seyler, W. Yao, and X. Xu, Valleytronics in 2D Materials, Nat. Rev. Mater. 1, 16055 (2016).
[52] See Supplemental Material at http://link.aps.org/ supplemental/10.1103/PhysRevX.12.011028 for more details on our alternating spin-momentum coupling models, three-dimensional antiferromagnetic junction models, and WannierBerri code first-principle calculations.

[53] C. W. Groth, M. Wimmer, A. R. Akhmerov, and X. Waintal, Kwant: A Software Package for Quantum Transport, New J. Phys. 16, 063065 (2014).

[54] G. Kresse and J. Furthmüller, Efficient Iterative Schemes for Ab Initio Total-Energy Calculations Using a Plane-Wave Basis Set, Phys. Rev. B 54, 11169 (1996).

[55] A. A. Mostofi, J. R. Yates, G. Pizzi, Y. S. Lee, I. Souza, D. Vanderbilt, and N. Marzari, An Updated Version of Wannier90: A Tool for Obtaining Maximally-Localised Wannier Functions, Comput. Phys. Commun. 185, 2309 (2014).

[56] S. S. Tsirkin, High Performance Wannier Interpolation of Berry Curvature and Related Quantities with WannierBerri Code, npj Comput. Mater. 7, 33 (2021).

[57] L. Smejkal, A. H. MacDonald, J. Sinova, S. Nakatsuji, and T. Jungwirth, Anomalous Hall Antiferromagnets, arXiv:2107 .03321 .

[58] E. V. Gomonay and V. M. Loktev, Spintronics of Antiferromagnetic Systems, Fiz. Nizk. Temp. 40, 22 (2014) [Low Temp. Phys. 40, 17 (2014)].

[59] D.-F. Shao, S.-H. Zhang, M. Li, and E. Y. Tsymbal, SpinNeutral Currents for Spintronics, Nat. Commun. 12, 7061 (2021). 